\title{
Simulating the emission of electromagnetic waves in the terahertz range by relativistic electron beams
}

\author{
J. I. Sakai ${ }^{1}$, Y. Nagasugi ${ }^{1}$, S. Saito ${ }^{1}$, and P. Kaufmann ${ }^{2}$ \\ ${ }^{1}$ Laboratory for Plasma Astrophysics, Faculty of Engineering, Toyama University, 3190 Gofuku, Toyama 930-8555, Japan \\ 2 Centro de Radio Astronomia e Astrofisica Mackenzie, Universidade Presbiteriana Mackenzie, 01302-907 São Paulo, SP, Brazil
}

Received 5 April 2006 / Accepted 23 May 2006

\section{ABSTRACT}

\begin{abstract}
Aims. We investigate the dynamics of relativistic electron beams propagating along a uniform magnetic field and the emission process of electromagnetic waves within the terahertz range from the solar photosphere. Our aim is to understand a new solar burst component emitting only in the terahertz range during the solar flare observed by Kaufmann et al. (2004).

Methods. We used a 2D3V fully relativistic electromagnetic particle-in-cell (PIC) simulation.

Results. We did three different kinds of simulations. The first simulation confirmed that the growth rate of relativistic electron beam instability agrees well with the theoretical estimation. From the second simulation of the electron beam with finite width, we found that the beams are confined along the magnetic field and the electromagnetic waves are generated forward of the electron beams. Some fraction of the electrons are accelerated more than the initial beam velocity. From the third simulation where the electron beams propagate into the high density region, we found that strong electromagnetic waves are generated backward to the electron beams. We also found that the higher frequency emission like $405 \mathrm{GHz}$, which originate in the strong magnetic field region, becomes stronger than the $212 \mathrm{GHz}$ emission, as shown in the observation by Kaufmann et al. (2004). These simulation results could be applied to the electromagnetic wave emission from the solar photosphere during the solar flares.
\end{abstract}

Key words. radiation mechanisms: general - plasmas - radiation mechanisms: non-thermal - methods: numerical Sun: radio radiation

\section{Introduction}

Recently, Kaufmann et al. (2004) reported the discovery of a new intense solar flare spectral radiation component, peaking somewhere in the shorter submillimeter to far-infrared range, identified during the large flare of November 4, 2003. The new solar submillimeter telescope (SST; Kaufmann et al. 2001), designed to extend the frequency range of solar flare observations to above $100 \mathrm{GHz}$, was used to detect this new component with increasing flux between 212 and $405 \mathrm{GHz}$ appearing along with, but separated from, the well-known microwave emission component. The novel emission component had three major peaks with time, originated in a compact source whose position remained within $15^{\prime \prime}$. Although the origin of the terahertz emission component during the flare is not known, it might be representative of emission caused by electrons with considerably higher energies than the energies assumed to explain solar microwave emissions producing incoherent synchrotron radiation (ISR) with maximum spectral emission somewhere in the $\mathrm{THz}$ range.

Recently, Kaufmann \& Raulin (2005) proposed a new interpretation of the intense microwaves observed at the same time, assuming that the accelerated ultrarelativistic electron beams undergoes microbunching instabilities producing broadband coherent synchrotron radiation (CSR) at microwaves, similar to what is observed in laboratory accelerators.

It is well known that most of the solar-burst radio emission occurs during flares and associated flare plasma dynamics. A flare-associated type III burst occurs at the impulsive phase, which is more intense at meter wavelengths and may have a continuum attached to it (see for reviews, Goldman et al. 1986;
Aschwanden 2004). It is believed that type III bursts are caused by mildly relativistic electrons $(\approx 10-100 \mathrm{keV})$ that are produced in impulsive solar flares, because bursts commonly occur in groups of ten or more, with a separation of seconds (Wild et al. 1963; McLean 1971). Recently Sakai et al. (2004) investigated the wave-emission process of solar type III radio bursts from magnetic reconnection region in a solar flare by using two dimensional, electromagnetic, relativistic particle-in-cell (PIC) code. They found that hot electron plasmas lead to the generation of Langmuir waves, and the generated Langmuir waves can be converted to the electromagnetic waves (solar type III radio bursts) through the linear direct-mode conversion process. It was also shown that the second harmonic emission is generated with time delay and at the amplitude is larger than for fundamental emission.

In the present paper we try to understand the new terahertz emission in terms of a plasma emission mechanism. We assumed that relativistic electron beams (REBs) are generated during strong solar flares, though the origin of the REBs is not known. Instead of studying mildly relativistic electrons for the solar type III bursts, we investigated the dynamics of relativistic electron beams propagating along a uniform magnetic field and the emission process of electromagnetic waves associated with the REBs. By using a 2D3V fully relativistic electromagnetic PIC simulation, we did three different kinds of simulations. In the first simulation we studied how the growth rate of relativistic electron beam instability agrees with the theoretical estimation. From the second simulation of the REBs with finite width, we studied how the REBs propagate along the magnetic field and how the electromagnetic waves are generated from the REBs. 


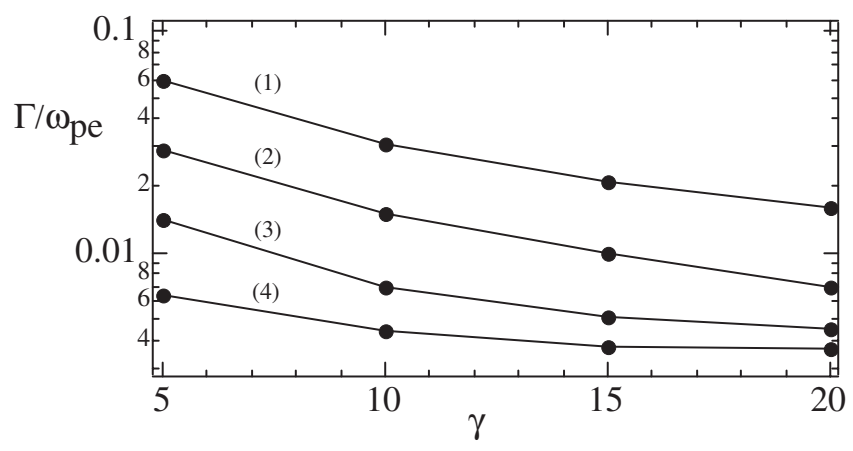

Fig. 1. The theoretical maximum growth rates $\Gamma$ normalized by $\omega_{\text {pe }}$ (vertical axis) vs. the electron-beam Lorenz factor $\gamma$ (horizontal axis) by changing the ratio between beam density $n_{\mathrm{b}}$ and background plasma density $n_{0}$ : (1) $n_{\mathrm{b}} / n_{0}=10^{-1}$, (2) $n_{\mathrm{b}} / n_{0}=10^{-2}$, (3) $n_{\mathrm{b}} / n_{0}=10^{-3}$, (4) $n_{\mathrm{b}} / n_{0}=10^{-4}$.

We found that some fraction of electrons are re-accelerated more than the initial beam velocity. From the third simulation where the REBs propagate into the high-density region corresponding to the solar photosphere, we found that strong electromagnetic waves are generated backward to the REBs. These simulation results could be applied to the electromagnetic wave emission with terahertz frequency range from the solar photosphere during the solar flares.

This paper is organized as follows. In Sect. 2 we investigate the dynamics of the REBs in uniform plasmas. In Sect. 3 we investigate the dynamics of the REBs with finite width. In Sect. 4 we investigate the dynamics of the REBs penetrating to the dense plasma. In Sect. 5 we summarize our results.

\section{Dynamics of REBs in uniform plasmas}

In this section we investigate the dynamics of the REBs propagating along the uniform magnetic field. It is well known that the REBs are unstable for the two-stream instability. The dispersion relation for the relativistic two-stream instability for the cold-beam approximation is given by the following expression,

$$
\frac{\omega_{\mathrm{pe}}^{2}}{\omega^{2}}+\frac{\omega_{\mathrm{pb}}^{2}}{\gamma^{3}\left(\omega-k v_{\mathrm{b}}\right)^{2}}=1,
$$

where $\gamma$ is the Lorenz factor that is given by $\gamma=[1-$ $\left.\left(v_{\mathrm{b}} / c\right)^{2}\right]^{-1 / 2}$ ( $c$ is light velocity and $v_{\mathrm{b}}$ is the beam velocity), $\omega_{\mathrm{pe}}=\sqrt{n_{0} e^{2} / \varepsilon_{0} m_{\mathrm{e}}}$, and $\omega_{\mathrm{pb}}=\sqrt{n_{\mathrm{b}} e^{2} / \varepsilon_{0} m_{\mathrm{e}}}$. Here, $n_{0}$ and $n_{\mathrm{b}}$ are background electron density and REB density, respectively. We solve the above dispersion relation by changing the ratio between $n_{0}$ and $n_{\mathrm{b}}$, as well as the beam velocity, and find the maximum growth rate for the REB instability. Figure 1 shows the summary of the obtained maximum growth rate normalized by $\omega_{\text {pe }}$ by changing the beam Lorenz $\gamma$ from 5 to 20 (horizontal axis). We also changed the ratio $n_{\mathrm{b}} / n_{0}$ from $10^{-1}$ to $10^{-4}$. As seen from Fig. 1 the growth rate of the REB instability tends to decrease when the beam velocity becomes higher and the ratio $n_{\mathrm{b}} / n_{0}$ decreases. In the realistic solar condition, the ratio $n_{\mathrm{b}} / n_{0}$ could be very small, as $n_{\mathrm{b}} / n_{0}=10^{-4}$. However, in the following PIC simulation, it is very hard to simulate the realistic ratio. Therefore we try to confirm the theoretical growth rate by the PIC simulation for the case of $n_{\mathrm{b}} / n_{0}=10^{-2}$.

We used $2 \mathrm{D} 3 \mathrm{~V}$, the fully relativistic electromagnetic PIC code, modified from the 3D3V TRISTAN code (Buneman 1993) for three different kinds of simulations. Here we investigate the first simulation of how the REBs propagate along the
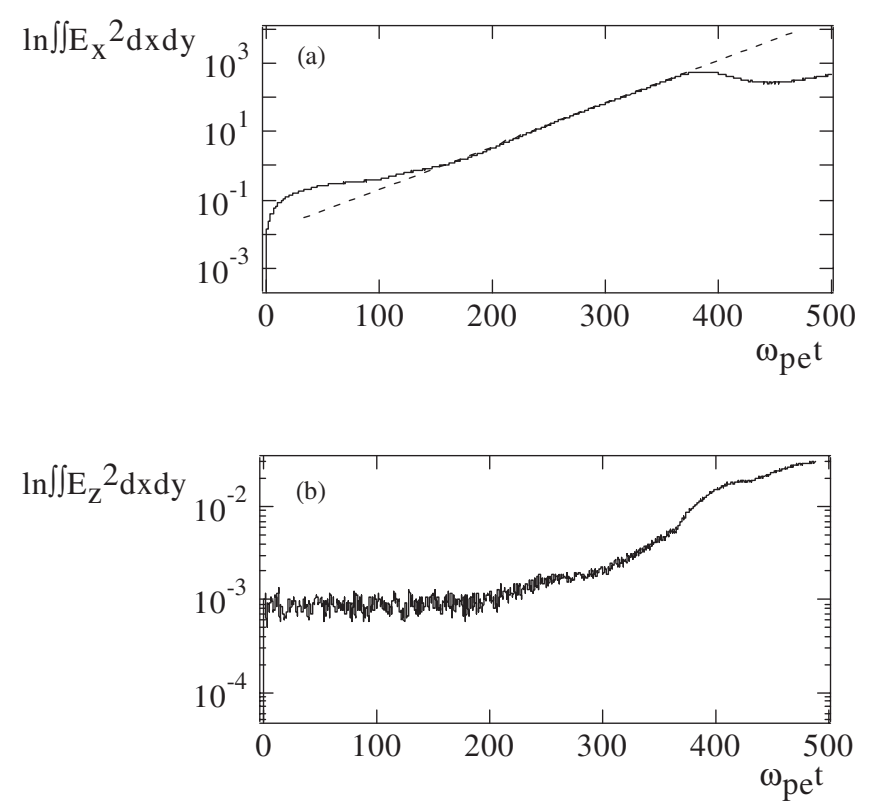

Fig. 2. a) Time development of the electrostatic field energy in the system $\iint E_{x}^{2} \mathrm{~d} x \mathrm{~d} y$, and b) the electric field energy in the system $\iint E_{z}^{2} \mathrm{~d} x \mathrm{~d} y$. The dashed line in a) is used to calculate the linear growth rate of the beam instability.

magnetic field in uniform plasmas. The system size is $L_{x}=$ $1024 \Delta$ and $L_{y}=6 \Delta$, where $\Delta(=1.0)$ is the grid size. The periodic boundary conditions for both $x$ - and $y$-directions are imposed on particles and fields. There are about 6.2 million electronion pairs, uniformly keeping the charge neutrality in the system. The average number of electron-ion pairs is 1000 per cell. The number density of the REBs is 10 per cell, and the number density of the background plasma is 990 per cell. The REBs propagate in the $x$-direction, parallel to the external uniform magnetic field. We investigated four cases by changing the beam Lorenz factor $\gamma=\left[1-\left(v_{\mathrm{d}} / c\right)^{2}\right]^{-1 / 2}(c$ is light velocity) to $5,10,15$, and 20 . The beam was assumed to be cold, $v_{\text {th,eb }}=0$, while the background electron thermal velocity is $v_{\text {th,e }}=0.1 c$. Other parameters are as follow: the time step $\omega_{\mathrm{pe}} \Delta t=0.05$, mass ratio $m_{\mathrm{i}} / m_{\mathrm{e}}=1836$, Debye length $v_{\mathrm{th}, \mathrm{e}} / \omega_{\mathrm{pe}}=1.0$, collision-less skin depth $\mathrm{c} / \omega_{\mathrm{pe}}=10 \Delta$. The initial electric field and electric current are equal to zero. Other physical quantities, such as the ratio of $\omega_{\text {ce }}$ to $\omega_{\text {pe }}$, plasma beta, Larmor radius $r_{\mathrm{L}}$, and Alfvén velocity $V_{\mathrm{A}}$, are $0.6,0.056,1.67 \Delta$, and $0.014 \mathrm{c}$, respectively.

Figure $2 \mathrm{a}$ shows the time development of the electrostatic field energy in the system. In the early stage, the electrostatic field energy shows the exponential growth that is calculated from the data to compare the theoretical estimation. Figure $2 \mathrm{~b}$ shows the time development of the electromagnetic field energy. The growth in the electromagnetic field energy shows the weak excitation of electromagnetic waves.

Figure $3 \mathrm{a}$ shows how the theoretical growth rate of the REB two-stream instability depends on the wave number, as well as the density ratio $n_{\mathrm{b}} / n_{0}$. As seen in the figure, the maximum growth rate is located near $k c / \omega_{\mathrm{pe}} \simeq 1$. By changing the beam velocity, we compare the theoretical maximum growth rate with the simulation results for $n_{\mathrm{b}} / n_{0}=10^{-2}$. Figure $3 \mathrm{~b}$ summarizes the comparison where the solid line shows the theoretical maximum growth rate, while the dashed line shows the simulation results. Except for the low-beam velocity case, we find good agreement between the theoretical values and simulation results. 

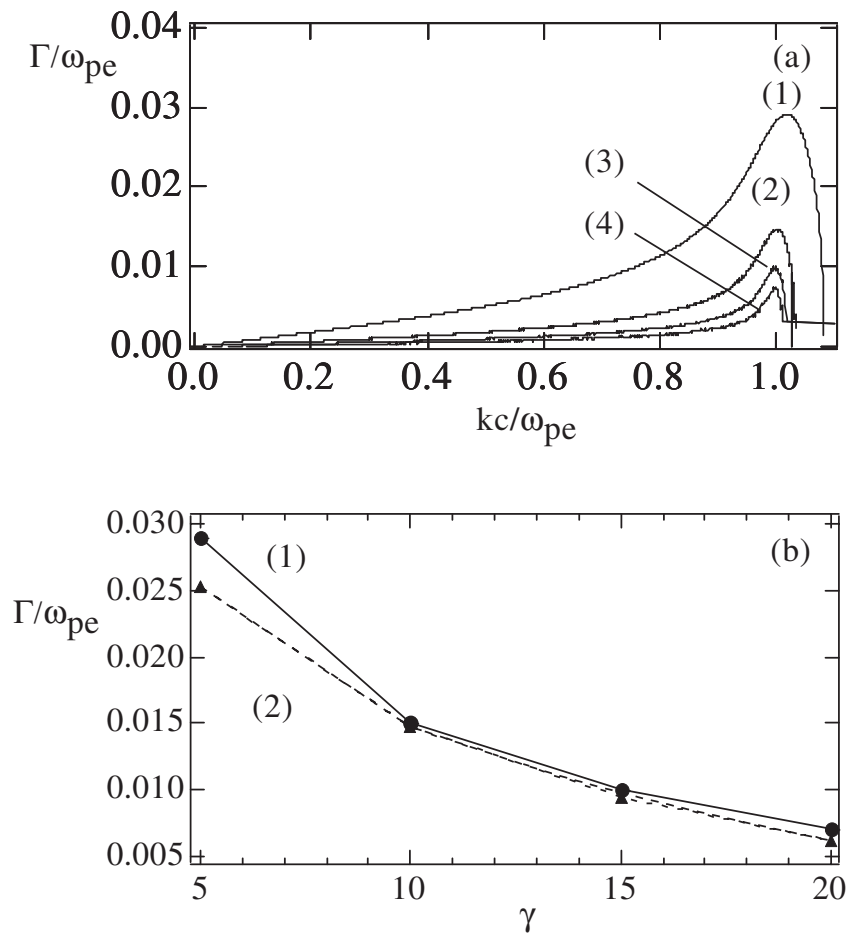

Fig. 3. a) The theoretical growth rates normalized by $\omega_{\text {pe }}$ (vertical axis) vs. the wave number $k$ normalized by $\omega_{\text {pe }} / c$ by changing the ratio between beam density $n_{\mathrm{b}}$ and background plasma density $n_{0}$ : (1) $n_{\mathrm{b}} / n_{0}=10^{-1}$, (2) $n_{\mathrm{b}} / n_{0}=10^{-2}$, (3) $n_{\mathrm{b}} / n_{0}=10^{-3}$, (4) $n_{\mathrm{b}} / n_{0}=$ $10^{-4}$. b) Comparison between the theoretical maximum growth rates ((1) solid line) and simulation results ((2) dashed line) for $n_{\mathrm{b}} / n_{0}=10^{-1}$, by changing the electron beam Lorenz factor from 5 to 20 .

\section{Dynamics of REBs with finite width}

In this section we study the dynamics of the REBs with finite width, focusing on how the REBs propagate along the magnetic field and the REBs are reaccelerated by using twodimensional system. The system size is $L_{x}=L_{y}=256 \Delta$. There are about 6.6 million electron-ion pairs, uniformly keeping the charge neutrality in the system. The average number of electron-ion pairs is 100 per cell. The system has beam and background electrons. Their number densities are 10 per cell for the beam and 90 per cell for the background electrons. The reason that the ratio $n_{\mathrm{b}} / n_{0}$ is $1 / 9$ in the present $2-\mathrm{D}$ simulation compared with the previous case is due to the limitation of computer power. The beam is parallel to the $x$-direction and the beam Lorenz factor is taken as $\gamma=\left[1-\left(v_{\mathrm{d}} / c\right)^{2}\right]^{-1 / 2}=5$. The REBs are confined with a finite width whose velocity is given by $v_{\text {beam }}=0.5 v_{\text {b }}[\tanh [(y-78) / 10]-\tanh [(y-178) / 10]]$. Other parameters are the same as in the previous simulations.

Figures $4 \mathrm{a}$ and $\mathrm{b}$ show the spacial distributions of the longitudinal electric field $E_{x}$ and electromagnetic component $E_{z}$ at $\omega_{\mathrm{pe}} t=320$. As seen in these figures, both electric fields are confined along the finite REBs. By using the above data, we perform space-time Fourier transformation to get the dispersion relations of the waves excited by the REBs. Figure 5a shows the dispersion relation of the Langmuir waves due to two-stream instability. As seen in Fig. 5b, showing the dispersion relation of excited electromagnetic waves, the electromagnetic waves are generated with almost the same frequency range as the Langmuir waves. The electromagnetic waves are excited in the same direction as the REBs. The solid curve in Fig. 5b shows the theoretical dispersion relation of the L-mode.

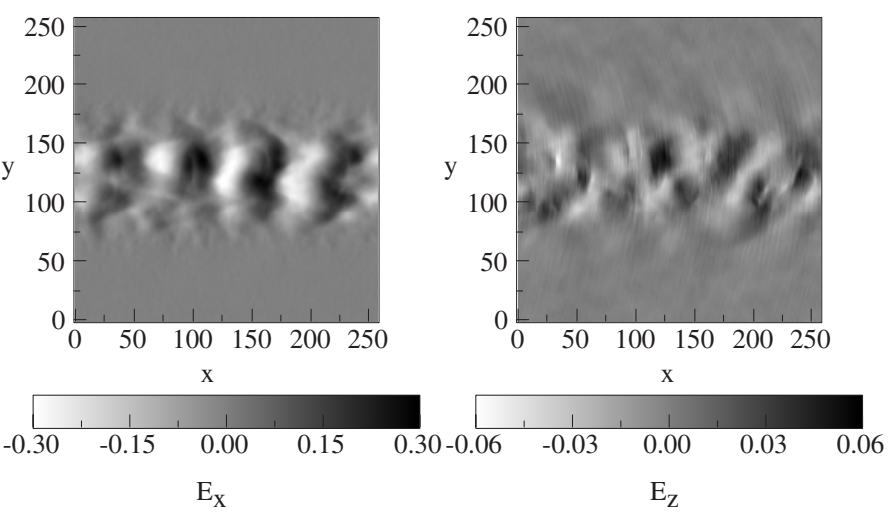

Fig. 4. The left figure shows the spacial distribution of the electric field $E_{x}$ corresponding to Langmuir waves. The right figure shows the spacial distribution of the electric field $E_{z}$ corresponding to emitted electromagnetic waves. Both data are taken at $\omega_{\text {pe }} t=320$.

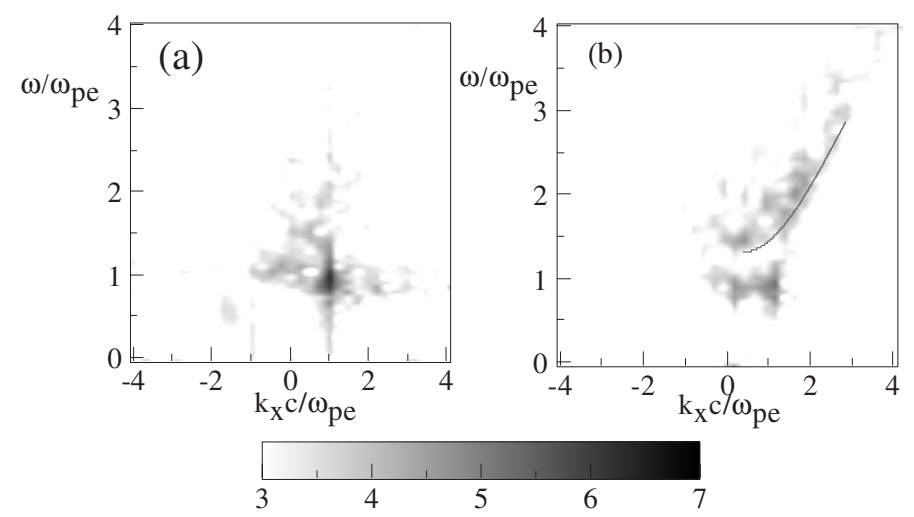

Fig. 5. a) The dispersion relation for the excited Langmuir waves and b) the dispersion relation for the emitted electromagnetic waves. The solid curve shows the theoretical dispersion relation of the L-mode.

Figure 6 shows the electron velocity distribution functions at (1) $\omega_{\mathrm{pe}} t=0$ and (2) $\omega_{\mathrm{pe}} t=500$. Figure 6a-1 is expanded from Fig. $6 \mathrm{a}$ in the high-velocity region. From Fig. 6a-1 we find that some fraction of the REBs are reaccelerated higher than the initial beam velocity.

From these simulation results, we may conclude that the REBs with finite width can propagate along the magnetic field and be reaccelerated. The electromagnetic waves are generated in the same direction as the REBs.

\section{Dynamics of REBs penetrating the dense plasma}

In this section we investigate what happens when the REBs propagate along the magnetic field and penetrate the high-density photosphere where the electron plasma frequency enters in the terahertz range because the electron density is about $10^{14}$. The system size taken in this simulation is $L_{x}=6000 \Delta$ and $L_{y}=$ $10 \Delta$. To simulate this high density region, we impose the density gradient in the simulation domain as $n_{\mathrm{e}}(x)=-0.141 x+900$. The REBs are imposed as $n_{\mathrm{b}}(x)=-5.5+\left(n_{\mathrm{b}} / n_{0}\right)(10 / 9)(1 .+1 . / \beta-$ $\left.1 /(4 \beta)\left(1-\tanh \left(\left(x-x_{\mathrm{c}}\right) / L\right)\right)^{2}\right)$, where $n_{\mathrm{b}} / n_{0}=0.05, \beta=1 / 8$, $L=100$, and $x_{\mathrm{c}}=5000$. The beam velocity is $v_{\mathrm{b}}=-0.9 c$ and the beam thermal velocity is $v_{\mathrm{tb}}=0.01 \mathrm{c}$, which propagates to the negative $x$-direction from the right side of the system. The assumption of uniform magnetic field might be reasonable, if the acceleration site is deeper in the active center, close to the photosphere, such as the path followed by the electrons, until reaching 

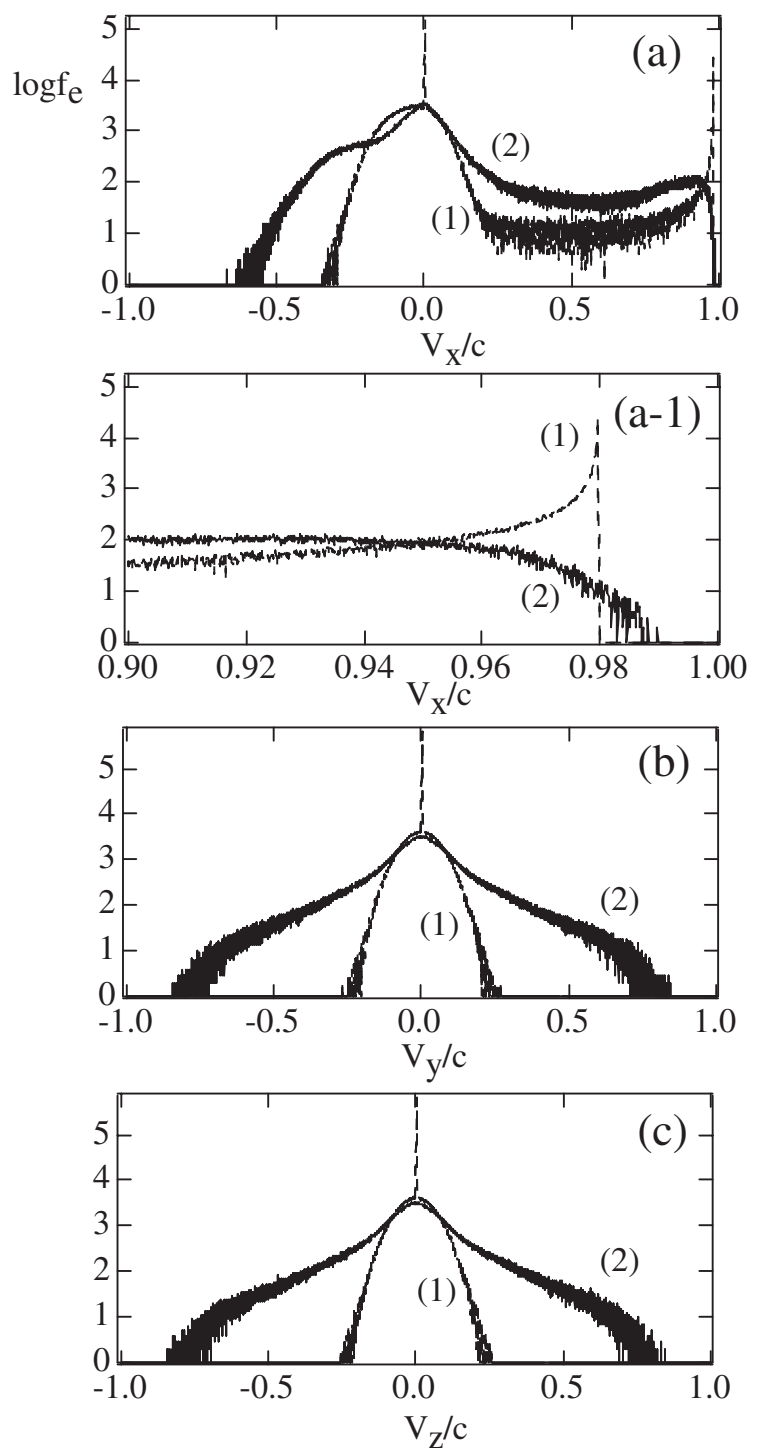

Fig. 6. The electron velocity distribution functions at (1) $\omega_{\mathrm{pe}} t=0$ and (2) $\omega_{\mathrm{pe}} t=500$.

denser regions is so short that there is no significant change in the magnetic field.

Figure 7 a shows the initial electron phase space, where the REBs are initially located in the region of $5000<x<6000$. As time goes on, the REBs penetrate to the high-density region, and some REBs are decelerated as seen in Fig. 7b, which shows the electron phase space at $\omega_{\text {pe }} t=500$.

Figure 8a shows the longitudinal electric field $E_{x}$ at $\omega_{\mathrm{pe}} t=$ 500, while Fig. 8 b shows the electromagnetic field component $E_{z}$ at $\omega_{\mathrm{pe}} t=500$. As seen from these figures, we found strong excitation on both electric fields. To find the characteristics of the excited waves, we performed time-and-space Fourier transformation for both electric fields with $395<\omega_{\mathrm{pe}} t<600$ and $1900<X<1900+4096$. Then we found the dispersion relations of excited waves that are shown in Fig. 9. Figure 9a shows the dispersion relation for the excited longitudinal waves. The strongest excitation is seen in the positive $x$-direction that is backward of the REBs. The strong emission of the electromagnetic waves is also observed backward of the REBs, as seen in Fig. 9b. Figure 9c shows the excited wave intensity $E_{z}$ (arbitrary in logarithmic scale) for positive $k c / \omega_{\text {pe }}=2.7$. The strong excitation is seen at about $\omega / \omega_{\mathrm{pe}}=3.0$. Therefore we conclude

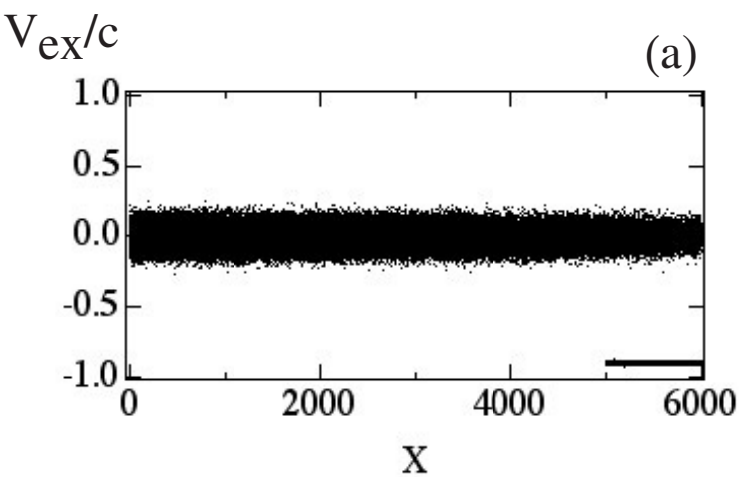

$\mathrm{V}_{\mathrm{ex}} / \mathrm{c}$

(b)

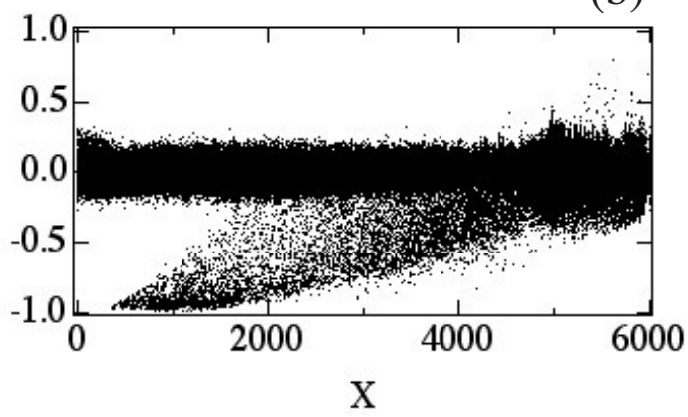

Fig. 7. The electron phase-space plots (the vertical axis is $V_{\mathrm{ex}} / c$ and the horizontal axis is $X$ ) at a) $\omega_{\mathrm{pe}} t=0$ and b) $\omega_{\mathrm{pe}} t=500$ for the initial electron beam velocity $v_{\mathrm{b}}=-0.9 c$.
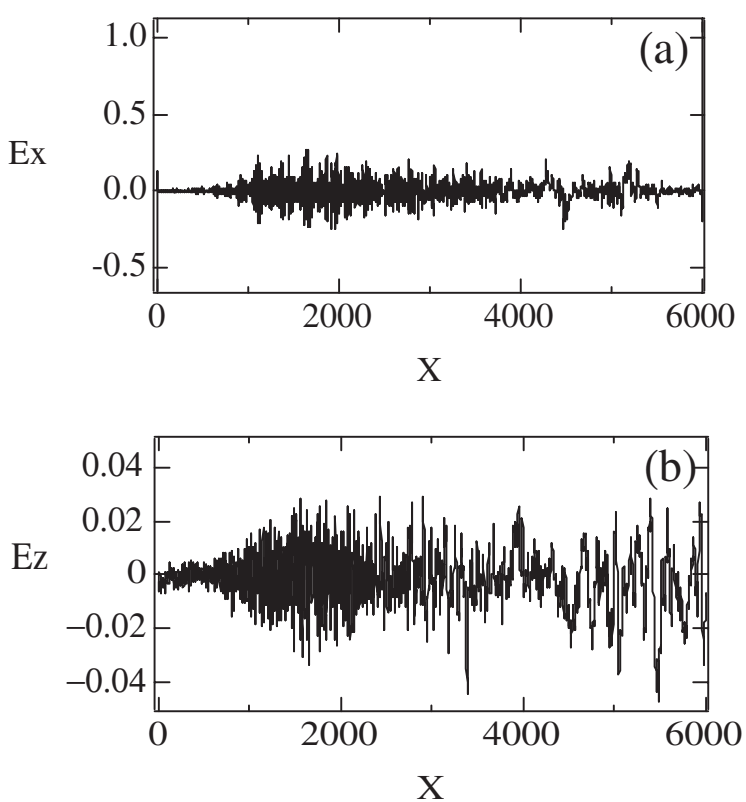

Fig. 8. a) The electric fields, $E_{x}$ and b) $E_{z}$ at $\omega_{\mathrm{pe}} t=500$.

that when the REBs penetrate the high-density photosphere from low-density corona region, strong backward-propagating electromagnetic waves are generated with the local electron plasma frequency, as well as $(2 \sim 3) \omega_{\text {pe }}$, a frequency that corresponds to the terahertz range. The emitted wave frequency of both the longitudinal and electromagnetic waves are in same frequency range; therefore, the conversion process from the longitudinal to the electromagnetic waves is a linear direct-mode conversion due to the density inhomogeneity. 
$\omega / \omega_{\mathrm{pe}}$

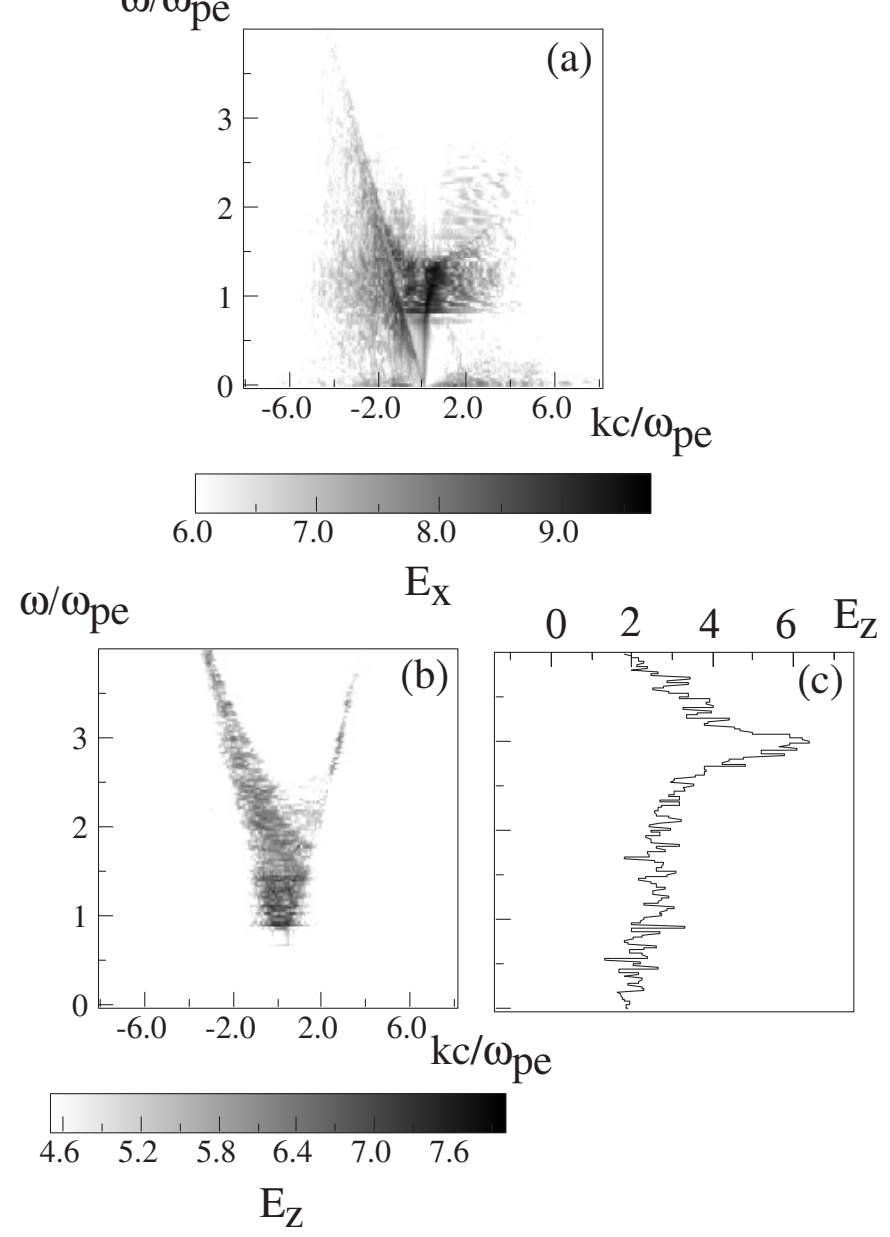

Fig. 9. a) The dispersion relation showing the excitation of Langmuir waves and $\mathbf{b}$ ) the dispersion relation of excited electromagnetic waves propagating backward of the REBs. Both dispersion relations are calculated from the field data of $395<\omega_{\mathrm{pe}} t<600$ and $1900<X<$ $1900+4096$. c) The wave intensity $E_{z}$ (arbitrary in logarithmic scale) at $k c / \omega_{\mathrm{pe}}=2.7$. The strongest emission occurs at $\omega / \omega_{\mathrm{pe}}=3.0$.

The observation by Kaufmann et al. (2004) shows that the $405 \mathrm{GHz}$ intensity is stronger than the $212 \mathrm{GHz}$ intensity. If the REBs penetrate the higher density region and stronger magnetic field region in the photosphere, is it possible for the higher frequency emission like $405 \mathrm{GHz}$ to become stronger than the $212 \mathrm{GHz}$ emission? To check this problem, we made the another simulation with about four times stronger $\left(\omega_{\mathrm{ce}} / \omega_{\mathrm{pe}}=1.72\right)$ than the previous magnetic field strength $\left(\omega_{\mathrm{ce}} / \omega_{\mathrm{pe}}=0.4\right)$. Except for the magnetic field strength, other parameters are kept as the same as in the previous case. The dispersion relation of the excited electromagnetic waves is shown in Fig. 10a, where both the L-mode and the R-mode are excited, but the L-mode intensity is stronger than the R-mode. In Fig. 10b, the excited wave intensity $E_{z}$ (arbitrary in logarithmic scale) for positive $k c / \omega_{\mathrm{pe}}=3.42$ is shown, where the strongest emission occurs at about $\omega / \omega_{\mathrm{pe}}=3.75$. If we compare Figs. $9 \mathrm{c}$ and $10 \mathrm{~b}$, we may conclude that the higher-frequency emission, like $405 \mathrm{GHz}$ that originates in a strong magnetic field region, becomes stronger than the $212 \mathrm{GHz}$ emission, as shown in the observation by Kaufmann et al. (2004). We really don't know how the electrons are injected with respect to the magnetic field direction. From the submm-wave observational standpoint, the results indicate that the impulsive emitting source remained stable in space within

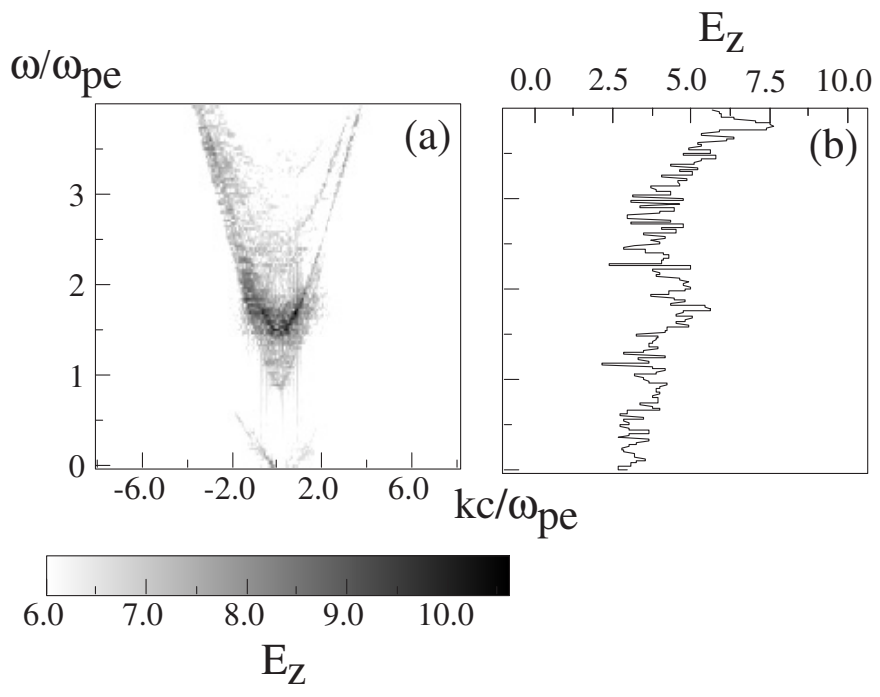

Fig. 10. a) The dispersion relation of excited electromagnetic waves propagating backward of the REBs. The dispersion relation is calculated from the field data of $400<\omega_{\mathrm{pe}} t<604.8$ and $1900<X<$ $1900+4096$. b) The emitted intensity $E_{z}$ (the horizontal axis unit is arbitrary in logarithmic scale) and the frequency (vertical axis) at $k c / \omega_{\mathrm{pe}}=3.42$ : the strongest emission occurs at about $\omega / \omega_{\mathrm{pe}}=3.75$.

the 15 arcsec. This result does not necessarily imply whether the emission was directional or not. However, the dependence of electromagnetic wave emission on the angle relative to the magnetic field direction will be investigated in future work.

\section{Summary}

We have investigated the dynamics of relativistic electron beams propagating along a uniform magnetic field and the emission process of electromagnetic waves within the terahertz range from the solar photosphere. The aim was to understand a new solar-burst component emitting only in the terahertz range during the solar flare observed by Kaufmann et al. (2004). By using a $2 \mathrm{D} 3 \mathrm{~V}$, fully relativistic electromagnetic particle-in-cell (PIC) simulation, we performed three different kinds of simulations. With the first simulation, we confirmed that the growth rate of the relativistic electron beam instability agrees well with the theoretical estimation. From the second simulation of the electron beam with finite width, we found that the beams are confined along the magnetic field and that the electromagnetic waves are generated forward from the electron beams. A fraction of the electrons are accelerated more than the initial beam velocity. From the third simulation where the electron beams propagate into the high-density region, we found that strong electromagnetic waves are generated backward from the electron beams. We also found that the higher-frequency emission - like $405 \mathrm{GHz}$ which originates in strong magnetic field region - becomes stronger than the $212 \mathrm{GHz}$ emission, as shown in the observation by Kaufmann et al. (2004). These simulation results could be applied to the electromagnetic wave emission from the solar photosphere during the solar flares.

The REB energies considered in these simulations, with Lorentz factor $=5$ (energies on the order of $2 \mathrm{MeV}$ ), produce synchrotron microwaves as they precipitate into the photosphere along magnetic fields of about 1000 Gauss, with a maximum spectrum at about $10-20 \mathrm{GHz}$, as observed. Further investigations are needed to better describe the spectral trends of electromagnetic waves generated from the photosphere by this 
mechanism. For higher REB energies, however, the synchrotron emission spectra maxima will shift towards higher frequencies, into the $\mathrm{THz}$ range, and may compete in intensity with the emission at photosphere levels produced by the process described in this paper. The relative importance of the two mechanisms should be investigated further.

Acknowledgements. We thank Dr. A. Posner for useful comments. One of the authors (Sakai) thanks P. Kaufmann for his hospitality during his stay in São Paulo. This work was supported by the exchange program between the JSPS and Brazil Academy of Science.

\section{References}

Aschwanden, M. J. 2004, in Physics of The Solar Corona (Springer-Verlag), Chap. 15,657
Budden, K. G. 1961, Radio Waves in the Ionosphere (New York: Cambridge Univ. Press)

Buneman, O. 1993, in Computer Space Plasma Physics, Simulation Techniques and Software, ed. H. Matsumoto, \& Y. Omura (Tokyo: Terra Scientific), 67

Goldman, M. V., \& Smith, D. F. 1986, Chap. 15 in Physics of the Sun, Vol. 2, ed. P. A. Sturrock, T. E. Holzer, D. M. Mihalas, \& R. K. Ulrich (D. Reidel Publ. Co.), 325

Kaufmann, P., \& Raulin, J.-P. 2005, Eos. Trans. AGU, 86(18), Jt. Assem. Suppl. Abstract SH54A-06

Kaufmann, P., et al. 2001, in Proc. SBMO/IEEE MTT-S Int. Microwave and Optoelctronics Cof., ed. J. T. Pinho, G. P. Santos Cavalcante, \& L. A. H. G. Oliveira (Piscataway: IEEE), 439

Kaufmann, P., Raulin, J.-P., Gimenez De Castro, C. G., et al. 2004, ApJ, 603, L121

McLean, D. J. 1971, Aust. J. Phys., 24, 201

Melrose, D. B. 1980, Aust. J. Phys., 33, 121

Sakai, J. I., Kitamoto, T., \& Saito, S. 2005, ApJ, 622, L157

Wild, J. P., Smerd, S. F. \& Weiss, A. A. 1963, ARA\&A, 1, 291 